\title{
NEUROPHYSIOLOGICAL ANALYSIS OF BRAIN-STIMULATION REWARD IN THE MONKEY
}

\author{
E. T. ROLLS, M. J. BURTON* and F. MORA** \\ University of Oxford, Department of Experimental Psychology, Oxford OX1 3UD (U.K.)
}

(Accepted October 18th, 1979)

Key words: brain-stimulation reward - orbitofrontal cortex - amygdala - substantia innominata — nucleus accumbens

\section{SUMMARY}

Neuronal activity related to brain-stimulation reward and to feeding was analyzed in rhesus monkeys and squirrel monkeys as follows. First, self-stimulation of the lateral hypothalamus, orbitofrontal cortex, amygdala and nucleus accumbens was found. Second, a population of single neurones in the lateral hypothalamus was found to be trans-synaptically activated from one or several self-stimulation sites. It was also found that populations of neurones in the orbitofrontal cortex and amygdala were activated from at least some of the self-stimulation sites. Thus, in the monkey, there is evidence for an interconnected set of self-stimulation sites, stimulation in any one of which may activate neurones in the other regions. These sites include the lateral hypothalamus, amygdala, and orbitofrontal cortex. Third, in one sample of 764 neurones in the lateral hypothalamus and substantia innominata which were activated from brainstimulation reward sites, $13.6 \%$ were also activated during feeding, by the sight and/or taste of food. The responses of the neurones with activity associated with taste occurred only while some substances (e.g. sweet substances such as glucose) were in the mouth, depended on the concentration of the substances being tasted, and were independent of mouth movements made by the monkeys. Fourth, the responses of these neurones occurred to food when the monkeys were hungry, but not when they were satiated. Fifth, self-stimulation occurred in the region of these neurones in the lateral hypothalamus and substantia innominata, and was attenuated by satiety. These results suggest that self-stimulation of some brain sites occurs because of activation of neurones in the lateral hypothalamus and substantia innominata activated by the sight

* University of Sussex, Laboratory of Experimental Psychology, Falmer, Brighton, Sussex.

** Departamento de Fisiologia, Facultad de Medicina, Universidad de Granada, GRANADA, Spain. 
and/or taste of food in the hungry animal, and that these neurones are involved in responses to food reward.

\section{INTRODUCTION}

Electrical stimulation of some areas of the brain is rewarding, in that the animals will work to obtain the stimulation. (As used in this paper, the term 'reward' simply means something for which the animal will work.) Although the areas of the rat brain which support self-stimulation or where electrical stimulation is negatively reinforcing have been extensively mapped ${ }^{14,16-18,23,26}$, only few studies have attempted a similar systematic examination of the primate brain ${ }^{2,19,33}$, although it is known that selfstimulation of some brain regions does occur in man ${ }^{1,4,34,35}$. Mainly in the rat, considerable effort has been devoted to identifying the particular neural pathways, for example, catecholamine pathways, which are best correlated with the areas where selfstimulation can be obtained (see ref. 6). However, only few studies have attempted to examine the neural systems which are actually activated by rewarding stimulation (see refs. 11,23 and 24). The study described here is the first in which the effects of rewarding electrical stimulation of the brain on neuronal activity in any forebrain region have been analyzed in the primate.

In this study of the neurophysiology of self-stimulation in the primate, electrodes were implanted in squirrel monkeys and rhesus monkeys at a variety of brain sites and tested for self-stimulation to determine which brain areas support self-stimulation in the monkey. Then the effects of the stimulation on neuronal activity in different brain regions were analyzed. The brain areas investigated in this study were chosen for one of the following reasons. Either the areas were homologous to those previously investigated by Rolls in the rat (hypothalamus, nucleus accumbens, and amygdala ${ }^{20-22}$ thus extending this work to the monkey, or they had been implicated in selfstimulation on the basis of fibre degeneration studies (orbitofrontal cortex, mediodorsal nucleus of the thalamus) ${ }^{\mathbf{3 3}}$.

When we had found self-stimulation sites in the monkey, and had been able to demonstrate populations of neurones in a number of different brain regions which were activated by the rewarding stimulation, the next question was the extent to which the activation of particular populations of these neurones might be related to the reward produced by the stimulation. To provide evidence on this, we next investigated what factors normally influence the activity of these neurons in the alert, behaving animal. Experiments are described which show that some of the neurons in the hypothalamus activated by brain-stimulation reward are also activated when a natural reinforcer, food, is given to the hungry monkey.

\section{METHODS}

Eight squirrel monkeys and two rhesus monkeys were implanted under pentobarbital sodium anaesthesia with arrays of monopolar stainless-steel stimulating 
electrodes insulated to within $0.3 \mathrm{~mm}$ of the tip as described elsewhere ${ }^{30}$. After a period of one week or more, the monkeys were placed in a primate chair and tested for self-stimulation using $0.3 \mathrm{sec}$ trains of $100 \mathrm{~Hz}, 0.1 \mathrm{msec}(0.5 \mathrm{msec}$ for the rhesus monkeys), square-wave, negative-going capacitatively-coupled pulses. The animals could turn on one train of stimulation each time they touched a brass bar placed at arm's length in front of them. If initial difficulty was experienced in shaping the animal to bar-press for $0.3 \mathrm{sec}$ trains, the stimulation was applied for as long as the animal touched the bar. This helped the monkeys to learn to self-stimulate. The animals were then shaped to respond with the $0.3 \mathrm{sec}$ trains. An alternative operant response which was easier for the monkeys to learn was licking a metal tube placed in front of the mouth. Initial training consisted of raising the stimulation current until a behavioural change was seen during priming, such as arousal. The current was then lowered and the animal was shaped until it learned to touch the bar to obtain brain stimulation, or failed to reach a criterion of 5 presses in $15 \mathrm{sec}$. In testing sessions repeated on different days the lowest current that would maintain self-stimulation was determined. The threshold was taken to be that current level at which less than 3 breaks of responding of more than $30 \mathrm{sec}$ occurred in any $3 \mathrm{~min}$ test period. After each break in responding an animal was given a $0.3 \mathrm{sec}$ train of priming stimulation. The threshold was measured as the current was both raised and lowered. When on three or more days the threshold currents were within $20 \%$ of each other and, at some arbitrary current usually 1.5 times threshold, the animal would sustain a stable self-stimulation rate over the 20 min test period, electrophysiological recording commenced.

It is worth noting that squirrel monkeys do not extinguish readily unless the response bar is placed at the limit of their reach. Under these conditions response rates are reduced and extinction occurs much more quickly.

The electrophysiological recording consisted of chronic recording from unanesthetized animals in eight squirrel monkeys and the two rhesus monkeys. The recordings of the activity of single neurons were carried out as previously described using tungsten microelectrodes ${ }^{30}$. These were slowly advanced through the brain towards the target structures. During this time single pulses, or multiple pulses identical to those which supported self-stimulation, were applied in turn to each of the selfstimulation electrodes to determine the effects of stimulation at the different reward sites on the activity of the cell being recorded. The pulses were delivered at the current which had supported self-stimulation or which had produced a behavioural change at electrodes negative for self-stimulation.

Activation of single cells by these stimulation pulses was noted for each electrode, and the activation was characterized as direct or trans-synaptic, as excitation or inhibition, and in terms of threshold for activation and latency of activation $^{20}$. A cell was classified as trans-synaptically driven if its action potential followed single stimulus pulses with variable latencies which were generally longer than those of directly driven units, in the range 2-100 msec (see Fig. 1). The shortest intrapair intervals of pulse pairs which trans-synaptically activated units would follow were usually in the range $1.5-10 \mathrm{msec}$. The latency of activation for trans-synaptic driving was taken as the shortest time at which changes in activation occurred with the 


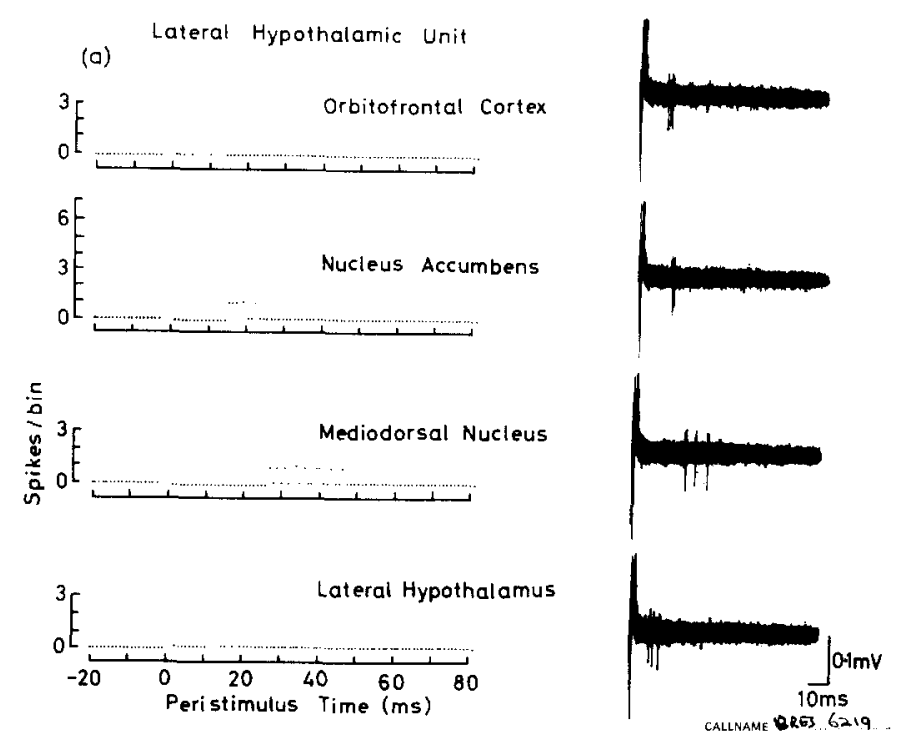

(b)
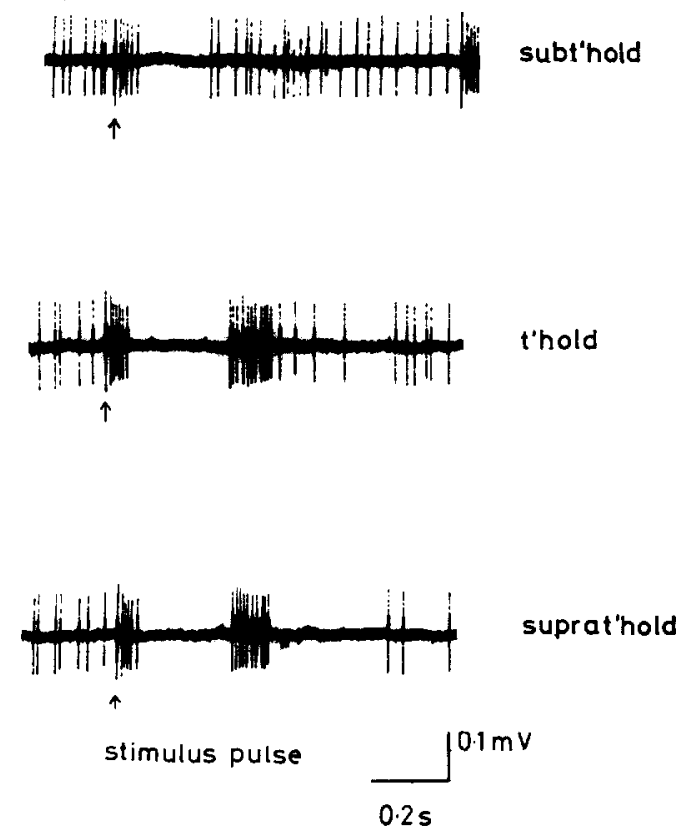

Fig. 1. a: example of a unit recorded in the lateral hypothalamus which was trans-synaptically activated (note the relatively long, variable latencies) by rewarding stimulation of the orbitofrontal cortex, nucleus accumbens, mediodorsal nucleus of the thalamus, and lateral hypothalamus. Left, peristimulus time histograms, bin width $=1 \mathrm{msec}, 5$ sweeps; right, phot ograph of unit, approximately 3 superimposed sweeps. b: excitation-inhibition produced by stimulation (arrow) at a self-stimulation site on the activity of a lateral hypothalamic unit in the squirrel monkey. At low currents (just below the current threshold for self-stimulation at the reward site-subt' hold, top trace) excitation lasting approximately $50 \mathrm{msec}$ was followed by inhibition lasting approximately $200 \mathrm{msec}$. At just higher currents (middle trace, at the threshold current for self-stimulation), this excitation-inhibition was followed by a further phase of excitation-inhibition, and this was more pronounced at higher currents (bottom trace) at a current supra-threshold for self-stimulation. 
stimulation current at the threshold for self-stimulation (see ref. 20 for details). The shape and magnitude of the action potentials indicated that the recordings were from cell bodies.

The factors which influenced the activity of hypothalamic neurons activated ty brain-stimulation reward were analyzed as follows. The monkey sat in a primate chair during the recording sessions, and could move his limbs freely, or have them moved by the investigator, and could eat and drink, so that any neuronal response associated with movement could be investigated. It was possible to investigate effects of somatosensory stimulation on the neurones using touch to the body or face. Effects of visual stimulation on the neurones were tested by presenting food and non-food objects to the monkey at a distance of $1 \mathrm{~m}$, as described in more detail elsewhere ${ }^{30,32}$. The monkeys were 4-18-h food deprived, and were willing to accept and eat food. When it was found that some of the neurons altered their activity when food was given to the monkey to eat, different tests as described below and elsewhere ${ }^{2,30}$ were used to determine whether the firing was associated with the sight of food, with the taste or smell of food, with the movements made to obtain food, or with general arousal. The activity of the neurones was measured when food and non-food odours were presented on cotton swabs, when the animal reached for and chewed edible and inedible objects, when the food was shown to the monkey, and to assess somatosensory effects, when the animal was touched, especially on the face, eyes, mouth, throat and stomach. For neurones whose activity altered while food was in the mouth, the neuronal activity was measured in the tests described above, and while the monkey's food (chow, oranges, bananas, dates, carrots and grapes), and standard volumes of test solutions (different concentrations of glucose, water and isotonic saline) were consumed. The solutions were useful in the separation of motor or somatosensory from gustatory effects, because the similar motor response, of drinking, was associated with different tastes.

The activity of neurones in the globus pallidus was also investigated in the same test situation and with the same procedures used for the neurones in the lateral hypothalamus and substantia innominata. This investigation was made in order to determine whether the tests used distinguished between neurones in different areas, and to investigate the activity of pallidal neurones during feeding in the monkey.

The sites where the neurones described in this paper were recorded were located in three different ways, using microlesions made through the recording microelectrodes, $\mathrm{X}$-rays taken on every track, and subsequent histological verification, as described fully elsewhere ${ }^{30,32}$.

\section{RESULTS}

\section{(1) Self-stimulation sites}

It was possible to ot tain good self-stimulation with electrodes in the caudal orbitofrontal cortex, nucleus accumbens, amygdala and in the lateral hypothalamus, preoptic area, and substantia innominata in the rhesus and squirrel monkeys. Examples of the self-stimulation sites in the squirrel monkeys, including also some in the mediodorsal nucleus of the thalamus, are shown in Fig. 2. Mapping with movable 

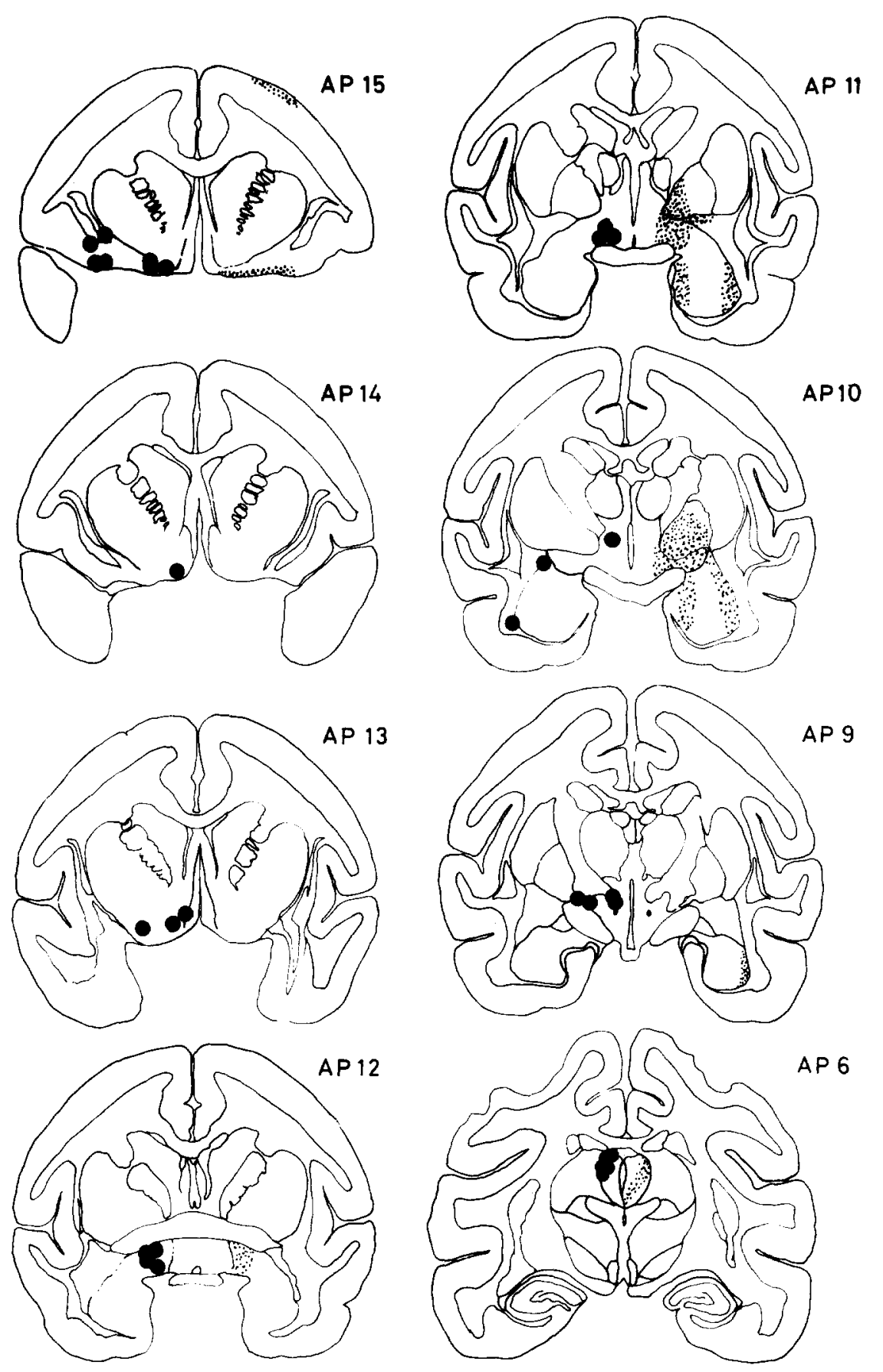

Fig. 2. Self-stimulation sites (filled circles) and recording sites (stipple) shown on coronal sections of the squirrel monkey brain at different distances ( $\mathrm{mm}$ ) anterior (AP) to the earbars. Orbitofrontal cortex sites: section at AP 15; nucleus accumbens stimulation sites: sections at AP 14, 13, and 12; different sites in the preoptic area, hypothalamus and substantia innominata: sections at AP 12,11, 10, and 9; mediodorsal nucleus of the thalamus sites: AP 6 . 

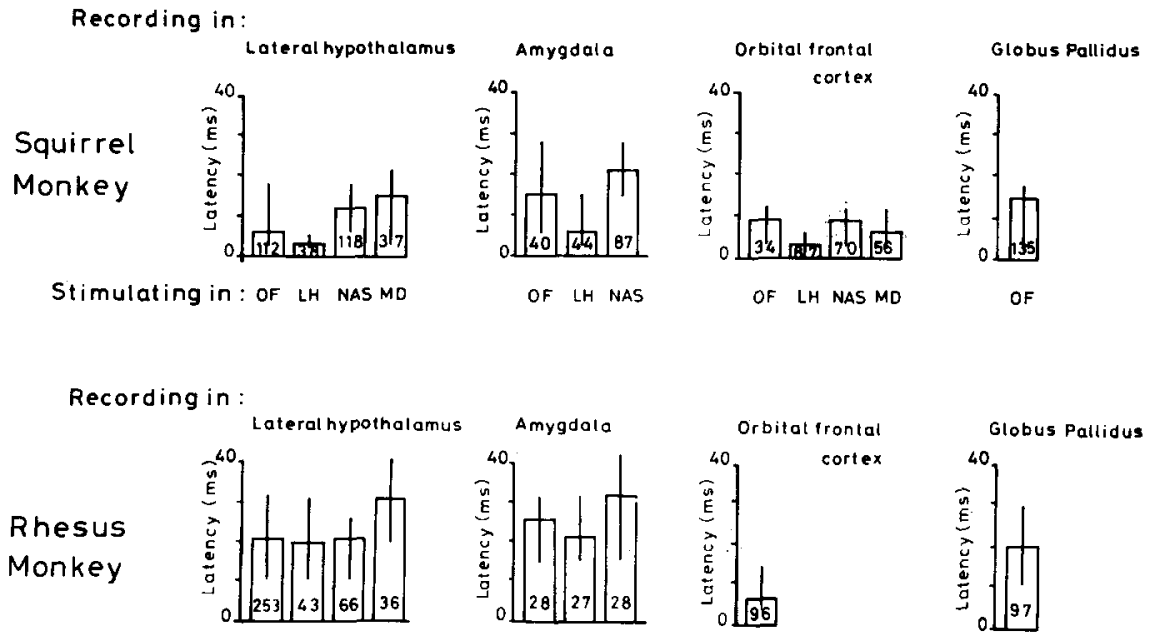

Stimulating in: OF LH NAS AM

OF LH Am

of

of

Fig. 3. Activation of cells recorded in different regions (lateral hypothalamus, etc.) from self-stimulation electrodes in different brain regions (OF, etc.) (OF, orbitofrontal cortex; $\mathrm{LH}$, lateral hypothalamus; NAS, nucleus accumbens septi; MD, mediodorsal nucleus of the thalamus; Am, amygdala). For each recording site the median latency and inter-quartile range of the activation from the different stimulation sites is shown. The numbers within each histogram show the number of instances of activation of a neuron, with each pair of stimulation and recording sites. The latencies measured were the shortest latencies from the stimulation at which excitation or inhibition was observed. In approximately $90 \%$ of the cases the effect measured was excitation. Histograms are only ploted if 10 or more instances of activation were found.

electrodes driven by a microdrive showed that the diencephalic area which supported self-stimulation ranged rostro-caudally from the level of the anterior commisure to the posterior hypothalamus, and extended laterally under the globus pallidus into the substantia innominata and dorsal amygdala.

\section{(2) Activation of neurones from self-stimulation sites}

Recordings were made from single neurones in a number of different brain sites from which self-stimulation could be obtained. A summary of the neuronal activation obtained in different recording sites from different self-stimulation sites is shown in Fig. 3. The activation recorded in these experiments was almost always trans-synaptic. It is clear, for example, that in the lateral hypothalamus, neurones were activated from self-stimulation sites in the orbitofrontal cortex, lateral hypothalamus itself, nucleus accumbens, and mediodorsal nucleus of the thalamus in the squirrel monkey, and from the orbitofrontal cortex, lateral hypothalamus itself, nucleus accumbens, and amygdala in the rhesus monkeys (Fig. 3). The area in which this activation occurred stretched laterally though the substantia innominata, and rostrally to the anterior commissure. Because, in any individual monkey positive self-stimulation electrodes were not present in all the brain areas tested at some time in the study, the numbers of instances of activation with a particular recording site/stimulation site combination cannot be compared across different combinations, but are included in the latency 
histograms to give an indication of the many hundreds of instances of activation observed in the course of this study, and of the reliable nature of the activation observed. Further, because this is the first study in which recordings have been made in any of these areas in relation to self-stimulation in the primate, any instances of activation observed in these areas were our primary interest. Because of this, and because of the limited durations of the recording sessions, exhaustive demonstrations that particular cells were not activated from self-stimulation sites were not attempted, and the percentage of possible instances of activation for a given electrode combination was not calculated. A second region in the primate in which neurons were activated from self-stimulation sites was the amygdala, as shown in Fig. 2. Activation of neurones in the basolateral and lateral amygdala was clearly demonstrated from the self-stimulation sites in the orbitofrontal cortex, lateral hypothalamus, and nucleus accumbens in the squirrel monkey, and from the orbitofrontal cortex, lateral hypothalamus and amygdala itself in the rhesus monkey. Recordings in the orbitofrontal cortex, mainly in the caudal orbitofrontal cortex area 13, in which self-stimulation was obtained, showed that neurones here were activated from self-stimulation sites in the lateral hypothalamus, nucleus accumbens and mediodorsal nucleus of the thalamus as well as from the orbitofrontal cortex itself in the squirrel monkey, and from the nucleus accumbens, lateral hypothalamus and amygdala (in limited numbers so that they are not represented in Fig. 3), as well as from the orbitofrontal cortex itself, in the rhesus monkey (Fig. 3). The results thus show that neurones in the hypothalamus, orbitofrontal cortex and amygdala are activated in self-stimulation of a number of different brain sites in the monkey. Activation of neurones recorded in the internal segment of the globus pallidus was found mainly from only one selfstimulation site, the orbitofrontal cortex, and self-stimulation in the globus pallidus was obtained only at a high current, which perhaps reflected current spread to the lateral hypothalamus and substantia innominata below, as the current required to maintain self-stimulation increased as the electrode was raised away from the hypothalamus.

\section{TABLE I}

Convergence of activation from different numbers of self-stimulation sites onto individual hypothalamic neurons

\begin{tabular}{|c|c|c|c|c|c|}
\hline \multirow[t]{2}{*}{ Number of neurons recorded in: } & & \multicolumn{4}{|c|}{ Number of self-stimulation sites } \\
\hline & & $0^{*}$ & $I$ & 2 & 3 \\
\hline \multirow[t]{2}{*}{ Lateral hypothalamus } & $\mathrm{r} * *$ & 10 & 93 & 91 & 15 \\
\hline & s & 4 & 173 & 114 & 45 \\
\hline \multirow[t]{2}{*}{ Amygdala } & $\mathbf{r}$ & 3 & 19 & 7 & 4 \\
\hline & s & & 115 & 36 & 5 \\
\hline \multirow[t]{2}{*}{ Orbitofrontal cortex } & $\mathrm{r}$ & & 79 & 3 & 1 \\
\hline & $\mathrm{s}$ & & 84 & 49 & 29 \\
\hline
\end{tabular}

* As described in the text, exhaustive tests for neurones for this column (i.e. hypothalamic neurones not activated from any self-stimulation site) were not made in this initial study.

** $\mathrm{r}=$ rhesus monkey, $\mathrm{s}=$ squirrel monkey. 
In many cases, particularly in the hypothalamus, in which instances of neuronal activation from a self-stimulation site were observed, it was found that an individual neuron was activated from a number of different self-stimulation sites. An example of this convergence of activation from self-stimulation electrodes in different sites onto an individual hypothalamic neuron is illustrated in Fig. 1a. A summary of the convergence from different brain regions onto individual neurons is shown in Table I. To allow the convergence in the different brain sites to be assessed, these results were drawn only from animals with 3 or more electrodes positive for self-stimulation and thus represent data from 3 squirrel monkeys and one of the two rhesus monkeys. It is clear that in both the rhesus and the squirrel monkey the effects of brain-stimulation reward of a number of different brain regions converge onto single hypothalamic neurons. This convergence was often from self-stimulation sites in the orbitofrontal cortex and nucleus accumbens, or from orbitofrontal cortex, nucleus accumbens and lateral hypothalamus, or from these three and the amygdala.

Although it was not a primary aim of this study, the first in which the activity of forebrain neurones in relation to brain-stimulation reward has been investigated in the primate, some evidence on the specificity of the neuronal activation from selfstimulation sites was obtained. This evidence is presented in the next two paragraphs.

In one rhesus monkey the electrode aimed at the nucleus accumbens septi (NAS) supported self-stimulation, and in the other monkey it did not, as it was too far ventral, although in this monkey behavioural signs of the stimulation, movements, were produced. It was thus possible to compare the numbers of neurones in the lateral hypothalamus activated from these two closely adjacent sites, and it was found that 65 hypothalamic neurones were activated from the electrode positive for self-stimulation and two from the electrode negative for self-stimulation. In the same two monkeys, the number of hypothalamic cells activated from the other electrodes were 112 and 159 , respectively, so that the small number of hypothalamic neurones activated from the NAS electrode which was negative for self-stimulation in the second monkey, was not simply because fewer tracks were made and fewer hypothalamic cells recorded in the second rhesus monkey. Thus for these two stimulation electrodes more hypothalamic cells were activated from the electrode which was positive for self-stimulation than from the electrode which did not support self-stimulation.

In three squirrel monkeys recordings were made in the dorsal frontal cortex, in the region stippled in Fig. 2, in order to compare the activation found here with the activation found in the other recording sites. It was found that although some neurones (57) in the dorsal frontal cortex were activated from the different selfstimulation sites used in the squirrel monkey, some dorsal frontal neurones (39) were also activated from non self-stimulation sites in these animals. For comparison, in these same three squirrel monkeys, 213 hypothalamic neurons were activated from the electrodes positive for self-stimulation, and 21 from the negative electrodes. These data suggest that relative to the dorsal frontal cortex, neurones in the lateral hypothalamus are more likely to be activated from self-stimulation than from nonself-stimulation electrodes positive for self-stimulation. Self-stimulation through the microelectrodes in the dorsal frontal cortex was tested, and was not obtained. 
It appears that in the monkey there is an interconnected system of sites activation of which is related to self-stimulation of any of these sites.

\section{(3) The response characteristics of neurones in the lateral hypothalamus and substantia} innominata activated by brain-stimulation reward

The responses of a sample of 764 hypothalamic neurones activated from at least one self-stimulation site as described above were analyzed in five behaving squirrel monkeys and two behaving rhesus monkeys, to provide evidence on the function of these activated neurones.

The responses of one category of these neurones which have activity related to the sight of food have been described elsewhere ${ }^{3,12,30,32}$. One other category of these neurones had responses which occurred during ingestion while food was in the mouth as described next. An analysis of the responses of one of the neurones during the ingestion of water and different concentrations of glucose solution is shown in Fig. 4. This neurone increased its firing rate above the spontaneous level when substances such as water or weak $(2.5 \%$ ) glucose were in the mouth, and showed a decrease below this level in firing rate which was closely related to the concentration of the glucose (see Fig. 4a). Other sweet solutions (such as blackcurrant juice) produced a comparable decrease in firing rate. Many other similar neurones did not respond to solutions such
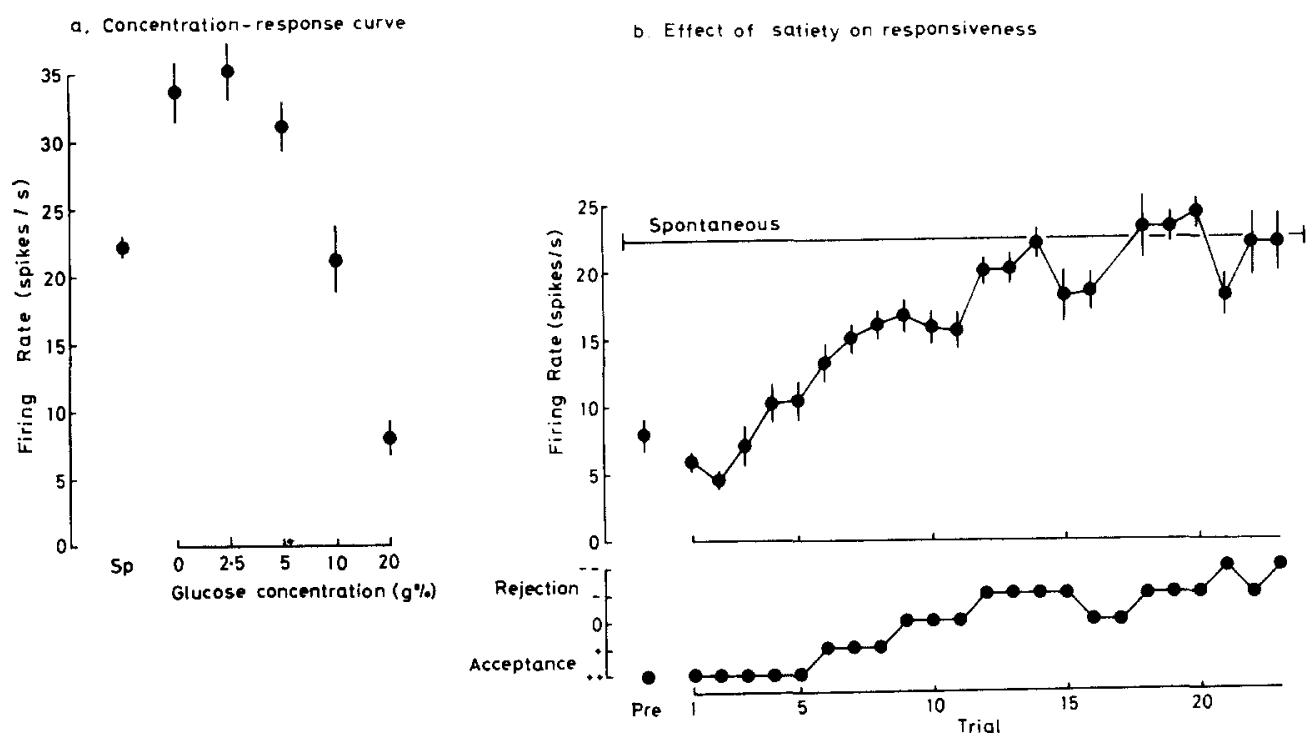

Fig. 4. a : effect of the glucose concentration $(w / v)$ tasted on the firing rate of a neurone in the rhesus monkey lateral hypothalamus. The firing rate (mean \pm S.E.M.) was measured in a $7-10$ sec period during which the monkey was drinking $2 \mathrm{ml}$ of the solution from a syringe. $0 \%$ represents drinking water. $b$ : effect of satiety on the response of the neuron shown in a. The firing rate was measured on each trial in a $7-10 \mathrm{sec}$ period in which the monkey drank $10 \mathrm{ml}$ of $20 \%$ glucose solution. The firing rate (mean \pm S.E.M.) decreased when the glucose was tasted on the early trials in which the monkey was still hungry, but as the monkey became satiated because of drinking the glucose (trials 5-15), the response when the glucose was tasted diminished to nil. Note that the spontaneous firing rate was not influenced by the transition to satiety. 
as water or saline by altering their firing rate from the spontaneous level, but did alter their firing rate only when some foods, e.g. sweet solutions, were in the mouth (see ref. 31). These responses to some but not other ingested solutions suggested that the responses of this category of hypothalamic neurones were associated with the taste of the food in the mouth, and this suggestion received support from the observations that these neurones were relatively uninfluenced by mouth movements and swallowing, or other face or body movements, or arousal.

Knowing from work on the rat that brain-stimulation reward of the lateral hypothalamus can be diminished by feeding the animal to satiety ${ }^{24}$, the effects of satiety on the responses of these hypothalamic neurones associated with the taste of food were next determined. An example is shown in Fig. 4b. This neurone, the same as that in Fig. 4a, decreased its firing rate from a spontaneous rate of 22 spikes/sec to 5-6 spikes/sec while $20 \%$ glucose was in the mouth at the start of the experiment, when the monkey was 8-20-h food deprived ('pre-satiety'). Then on consecutive trials (separated by $30 \mathrm{sec}$ ) the monkey was fed $10 \mathrm{ml}$ of the $20 \%$ glucose solution, and the response of the neurone which occurred while the glucose was in the mouth being ingested was measured. Gradually, particularly between trials 4 and 12 , the response of the neurone to the glucose became less (i.e. it changed its firing rate by a smaller amount from the baseline level), until by trial 18 , when the monkey was satiated, ingestion of the glucose did not produce any response in the neurone. For comparison, the satiety produced by the glucose, measured by whether the monkey accepted or attempted to reject the glucose, is shown below. (The glucose was delivered into the monkey's mouth, and he swallowed it, even when he was showing satiety by attempted rejection, to ensure standard testing conditions.) It is clear that the neurone only responded in association with the taste of the glucose when the monkey was hungry, and did not respond when the monkey was satiated. It should be noted that only the responsiveness, and not the spontaneous firing of the neurone, was influenced by the change from hunger to satiety. This modulation of reponsiveness by hunger was found in all the units tested (see ref. 3).

Responses of this type associated with the taste of food were found in 33 neurones $(4.3 \%$ of the sample). Twenty-one responded with an increase of firing rate, and 17 with a decrease. (Nineteen of these neurons responded to both the sight, as described below, and the taste of food.) The effects of satiety on the responses of these neurones associated with the taste of food has been confirmed five times in five different neurones recorded in three squirrel monkeys and two rhesus monkeys (see also ref. 3).

Of the other neurons in this sample of 764 hypothalamic neurones activated by brain-stimulation reward whose responses were analyzed in the behaving animal, it was found that $71(9 \%)$ had responses which occurred immediately before the animal was fed, in association with the sight of food. The response characteristics of these neurones have been described elsewhere ${ }^{30}$. It has been found that these neurones, too, only respond to food when the monkey is hungry3. Further, the responses of these neurones become associated with the sight of food during learning ${ }^{12}$, and fire with latencies of 150-200 msec in a visual discrimination for food, so that they precede, and predict, the responses of the hungry monkey to food ${ }^{32}$. 
TABLE II

Convergence of activation from different numbers of self-stimulation sites onto hypothalamic neurons which had or did not have food-related responses

Number of self-stimulation sites

$\begin{array}{lll}0 & 1 & 2\end{array}$

$\left.\begin{array}{llrrrr}1 \text { Rhesus monkey } & \text { Feeding-related } & 0 & 7 & 14 & 6 \\ & \text { Not feeding-related } & 10 & 86 & 77 & 9\end{array}\right\} \chi^{2}=5.7, \mathrm{df}=1, P<0.02$

Of the remaining hypothalamic neurones, another group had activity which was associated with arousal and general body movement, with mouth, face or arm movements, with visual stimulation by moving objects, or with the sight of aversive visual stimuli, as described elsewhere ${ }^{27,32}$, and 128 neurones $(16.8 \%)$ were classified after analysis in this category. Many neurones $(69.6 \%$ of this sample) were unaffected by the above types of stimulation or by movement or by feeding, or had small responses or could not be analyzed sufficiently to enable them to be classified into the above categories. Thus of the sample of 764 hypothalamic neurones activated by brain-stimulation reward and analyzed during behaviour, 104 (i.e. $71+33$ ), or $13.6 \%$ had responses associated with the sight and/or taste of food, and the responsiveness of these classes of neurones depended on hunger. It should be emphasized that these neurones were found in the lateral hypothalamus, and in an area stretching laterally into the substantia innominata, and rostrally through the preoptic area to the anterior commissure (see also examples in refs. 30 and 32). In relation to brain-stimulation reward, it was found that neurones activated from many self-stimulation sites were particularly likely to be activated by the sight and/or taste of food (see Table II). (As in

Fig. 5. a: hypothalamic units activated by both brain-stimulation reward and by natural reward (the sight of food) were recorded at the base of this microelectrode track. Self-stimulation through the recording microelectrode occurred in the region of these units as shown in the histogram of the current threshold for self-stimulation through the recording microelectrode. Both the response of the units to food and the self-stimulation in this region were attenuated if the animal was not hungry. Above this region in the globus pallidus, some activation of units, particularly from reward sites in the orbitofrontal cortex and mediodorsal nucleus of the thalamus, occurred, but the firing of these units occurred in relation to head movements, and self-stimulation of this region was poor (it probably occurred because of current spread to the hypothalamus below). The activation of the units was trans-synaptic (S) or direct (D) from self-stimulation sites in the orbitofrontal cortex (OF), nucleus accumbens (NA), mediodorsal nucleus of the thalamus (MD) or lateral hypothalamus (hl) with the latencies shown in msec. Abbreviations: Am, amygdala ; $\mathrm{Ca}$, caudate nucleus; $\mathrm{CC}$, corpus callosum; $\mathrm{GP}$, globus pallidus; IC, internal capsule; Lh, lateral hypothalamus; OC, optic chiasm; Put, putamen; SI, substantia innominata; th, thalamus; $V$, ventricle. $b$ : the mean of the self-stimulation threshold current $(\mathrm{mA})$ as a function of the depth $(\mathrm{mm})$ of the microelectrode in the brain for eight separate experiments in the squirrel monkeys. The lowest thresholds in the 8 experiments were found at $18-20 \mathrm{~mm}$ below the dura, i.e. at the level of the lateral hypothalamus and substantia innominata. The stimulation $(0.1 \mathrm{msec}$ pulses at $100 \mathrm{~Hz}$ lasting for $0.3 \mathrm{sec}$ ) was applied through the recording microelectrode after recording had finished as the microelectrode was repeatedly raised and lowered to different depths. 

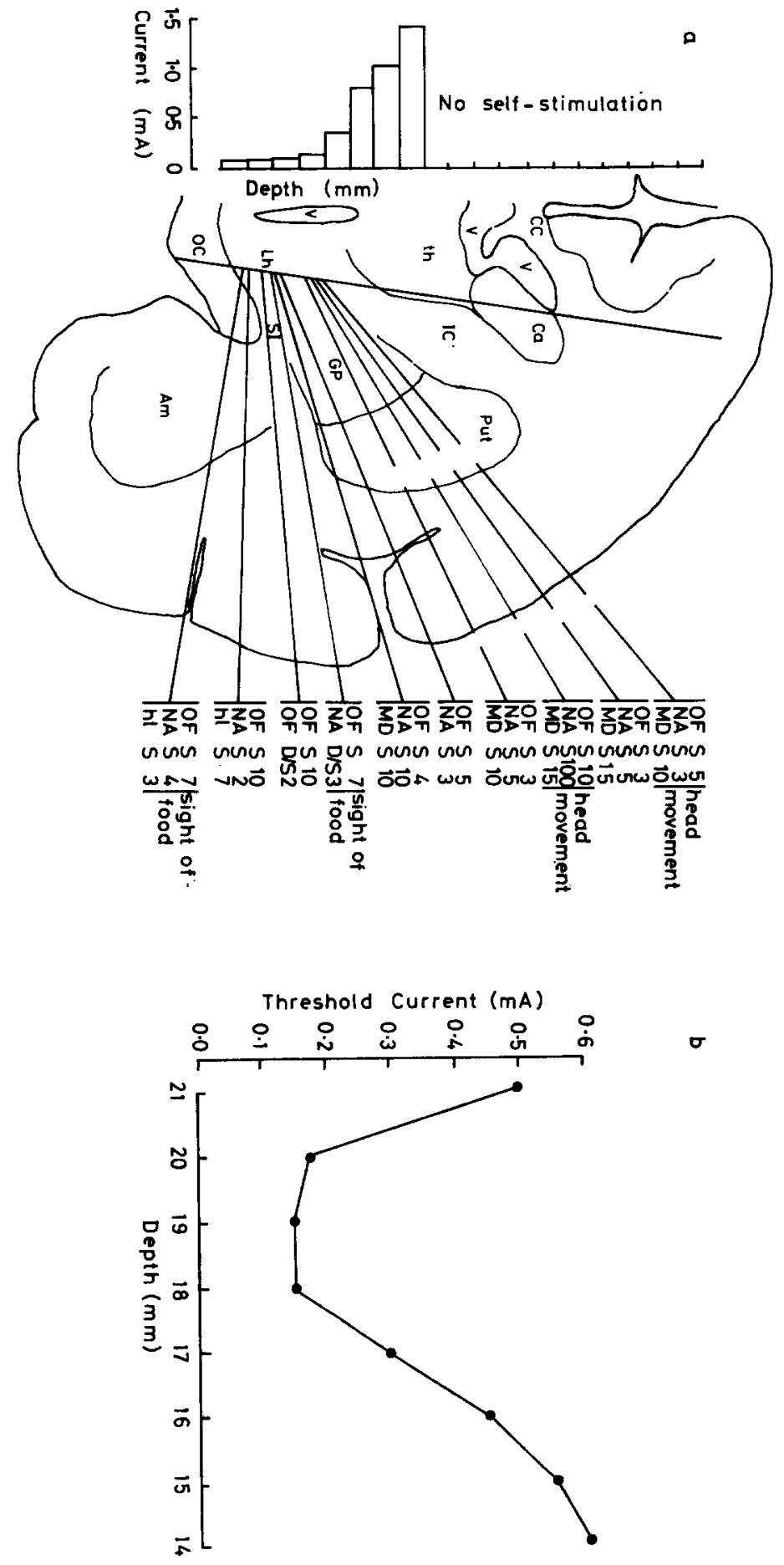
the case of Table 1 the data are drawn only from the animals with three or more electrodes positive for self-stimulation.) This suggests a close relation between the effects of the sight and taste of food and brain-stimulation reward on these neurones.

(4) Effects of stimulation in the region of the neurones in the lateral hypothalamus and substantia innominata

Electrical stimulation was applied through the recording microelectrode after recordings had been completed on some of the tracks. It was found that selfstimulation occurred when the microelectrode was in the region of the neurones which were activated by brain-stimulation reward and had responses associated with food. The current threshold for the self-stimulation with $0.1 \mathrm{msec}$ pulses was usually low $(100 \mu \mathrm{A})$ in the region of these neurones, and as the microelectrode was moved away from this region the threshold for self-stimulation increased, until finally selfstimulation would not occur at any current, and usually only motor movements were elicited. An example of one of these experiments is shown in Fig. 5a. It is shown in Fig. $5 b$ that in 8 experiments in the squirrel monkey in which stimulation was applied through the recording electrode at different depths, self-stimulation was obtained at low currents at depths of $18-20 \mathrm{~mm}$ (corresponding to the depth of the lateral hypothalamus), and higher currents had to be given to obtain self-stimulation as the microelectrode was moved away from this region. In these experiments care was taken to ensure that the measured self-stimulation threshold at a particular electrode depth was constant, as the initial effect of stimulation through the varnished electrode was to lower the impedance of the electrode. Although not usually tested, it was noted in some tracks that eating or other stimulus-bound behaviour might be elicited by $30 \mathrm{sec}$ trains of electrical stimulation.

\section{(5) Effects of satiety on the self-stimulation in this region}

As it was known that the responses of neurones in this region associated with food rewards are decreased if the monkey is fed to satiety ${ }^{3}$, the effects of satiety on brain-stimulation reward in this region were determined. In experiments in the squirrel monkey it was found that the self-stimulation rate through the microelectrode could sometimes be decreased, and the current required to maintain self-stimulation be increased, by feeding the monkey to satiety with $20 \%$ glucose solution (Fig. 6). The effect did not appear to be due to a general depression of behaviour, for selfstimulation at some other sites (for example in the nucleus accumbens) was not affected by satiety.

\section{(6) Activity during feeding and drinking of neurones in the globus pallidus}

A number of units were found in the globus pallidus which altered their activity during ingestion. However, the characteristics of these units were different from those of the units in the lateral hypothalamus and substantia innominata. An example of the responses of one of the pallidal units has been shown in Fig. 2 of ref. 31, which shows that the firing rate of the unit increased to more than $60 \mathrm{spikes} / \mathrm{sec}$ when any fluid was swallowed. Thus the activity of the unit appeared to be related to ingestion or 


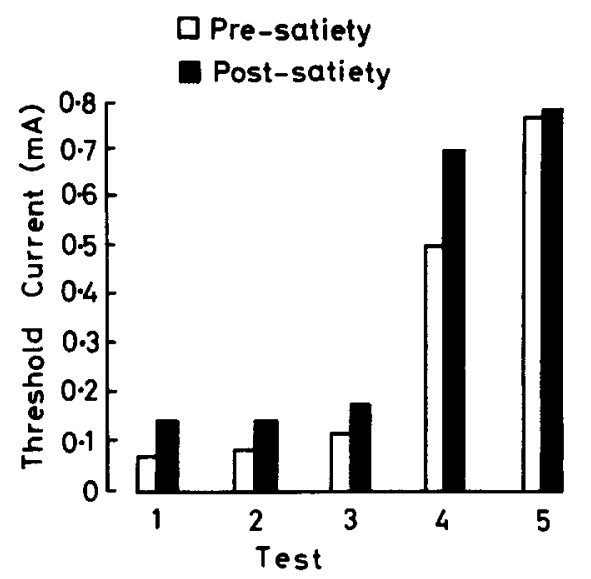

Fig. 6. The histogram shows the threshold current for self-stimulation obtained before (pre-satiety) and after (post-satiety) satiating the squirrel monkeys with $20 \%$ glucose solution. The self-stimulation measured was that obtained through the microelectrode when it was in the region of the lateral hypothalamus and substantia innominata after a recording track had been made. Results obtained for 5 tests on different tracks (usually in different monkeys) are shown.

swallowing per se. Several units of this type were found. Other units responded when the lips were touched, or when air was blown onto the lips, or when the monkey moved his tongue or chewed, or moved his head, or when the monkey reached (see refs. 3, 27 and 30). Thus although the firing pattern of these units was related to ingestion, the responses were obtained with a wide variety of substances ingested and thus could be differentiated from the responses of hypothalamic neurones associated with the taste of food.

Units in the globus pallidus often fired with high maximal firing rates between 50 and $100 \mathrm{spikes} / \mathrm{sec}$. This characteristic also helped to differentiate these neurones from the neurones in the hypothalamus and substantia innominata below the globus pallidus. Further, the responsiveness of pallidal units was found to be independent of whether the animal was hungry or satiated ${ }^{3}$.

\section{DISCUSSION}

Good self-stimulation sites in the monkey were found in the caudal orbitofrontal cortex, mediodorsal nucleus of the thalamus, nucleus accumbens septi, lateral hypothalamus and substantia innominata, and in the basolateral amygdala and the dorsal amygdala. These findings are consistent with and extend the findings of Briese and Olds ${ }^{2}$, Routtenberg, Gardner and Huang ${ }^{33}$, Plotnik et al. ${ }^{19}$ and German and Fetz?.

A main finding of this study was that neurones in the caudal orbitofrontal cortex, lateral hypothalamus, substantia innominata, and amygdala were activated by stimulation applied to these self-stimulation sites or to self-stimulation sites in the nucleus accumbens (see Fig. 3). Often, and particularly in the lateral hypothalamus and substantia innominata, individual neurones were activated from several of these 
different self-stimulation sites (see Table I). Thus in the monkey there is a highly interconnected set of self-stimulation sites in which stimulation at one site activates neurons in the other self-stimulation sites.

Although the prinary aim of this initial study was to identify forebrain areas in which neurones were activated by rewarding stimulation, some evidence was obtained on the specificity of the type of activation which was found. First, when recordings were made in the internal segment of the globus pallidus, it was found that if neurones were activated during self-stimulation, the main site from which activation occurred was the orbitofrontal cortex (see Fig. 3). When self-stimulation of the globus pallidus was tested with a roving microelectrode it was found that the further the microelectrode was raised above the substantia innominata, the higher was the current needed to maintain self-stimulation. Thus either the activation of pallidal neurones only occurred at high currents, or the self-stimulation occurred by current spread towards the substantia innominata. When the activity of pallidal neurones was recorded during behaviour rewarded by food, it was found that neuronal activity related to the task occurred during movements, rather than in relation to the presentation of food (see also ref. 30). Thus, although activation of neurones in this extrapyramidal structure occurred, it was mainly from one self-stimulation site, the activity of these neurones was related to movements, and it was not clear whether selfstimulation of this region occurred. Second, when recordings were made in the lateral hypothalamus and substantia innominata of the rhesus monkeys, it was found that neurones were more likely to be activated from a nucleus accumbens electrode which supported self-stimulation than from one at which stimulation elicited effects other than self-stimulation. Third, in the squirrel monkey's dorso-lateral frontal cortex, although some neurones were activated from reward sites, neurones were also activated from non-reward sites, and self-stimulation was not obtained in this region. This region thus provided a contrast to the lateral hypothalamus, in which neurones were more likely to be activated from reward sites than from non-reward sites, and in which self-stimulation was obtained. These comparisons emphasize that it is not to be expected that electrical stimulation of the brain which provides reward excites only one neural system. Rather it is to be expected that several or many systems will be activated, with activation of some of the systems being related to the positively reinforcing effects produced, while activation of other systems may be related to side effects such as minor motor movements. Further, it is possible that stimulation at nonreward sites activates neurones normally involved in brain-stimulation reward, but that concomitant activation of another system is incompatible with self-stimulation. In this situation it is necessary to analyze systems activated from reward and non-reward electrodes and to test for self-stimulation in these systems, and also to analyze the function of neurons activated from self-stimulation sites.

When the responses of a sample of 764 neurons activated from self-stimulation sites were analyzed, it was found that 104 neurons (13.6\% of the sample) had activity associated with the sight and/or taste of food. Some of the neurones fired during the ingestion of certain foods or fluids (as described here), and others fired before feeding, when the monkeys were looking at food ${ }^{30}$. Both types of response associated with food 
only occurred in the hungry animal, i.e. when the food was rewarding, as indicated by the animal working to obtain and accepting the food ${ }^{3,12}$. Thus these neurones are activated when either brain-stimulation reward of some sites, or food reward, is given to the animal. (It may be noted that as both excitation and inhibition of an individual neurone may be produced by the electrical stimulation, it is not easy to relate the direction of the effect on neuronal firing rate of food - an increase or a decrease - to whether the electrical stimulation excited or inhibited the neurone. The main point which can be made is that the neurones described receive an input from both food reward and brain-stimulation reward.) Further, electrical stimulation in the region of those neurons was found to be rewarding (see above). It was also shown that at some of these sites, the self-stimulation rate was reduced if the animal was satiated.

These findings indicate a close relationship between food reward, brain-stimulation reward, and the activation of neurones in the lateral hypothalamus and substantia innominata. A comparable relationship may also exist in the rat hypothalamus, in which some neurones alter their activity during feeding ${ }^{9}$, in which some neurones are affected by food-related gustatory stimuli, particularly if the rat is hungry ${ }^{14}$, and near which neurones self-stimulation is likely to occur.

The findings that some neurones in the lateral hypothalamus and substantia innominata altered their activity during the ingestion of certain foods or fluids, and that their activity was not affected by movements of, for example, the face and mouth, are consistent with the possibility that the responses of these neurones are gustatory. Further evidence consistent with this possibility is that in the test situation used the responses of these neurones can be differentiated from the responses of other neurones in the same area and of neurones in the globus pallidus (see above) and substantia nigra $^{13}$, which have activity which appears to be related to motor responses. For example, some neurones in these areas had activity which altered during feeding, but showed similar changes in activity when different fluids were ingested, and had activity which could be related to identifiable face, mouth or throat movements. It is of interest that in the lateral hypothalamus and substantia innominata of the alert monkey neurones with responses which appear to be gustatory are recorded, for in the lateral hypothalamus of the anesthetized rat neurones with gustatory responses have been described ${ }^{\mathbf{1 4}}$ and, also in the rat, gustatory pathways have been traced from the nucleus of the solitary tract to the pontine taste area, and from there to the central nucleus of the amygdala, lateral hypothalamus, and substantia innominata, as well as to the thalamic taste relay ${ }^{15}$. It is also of interest that at the stage of the lateral hypothalamus and substantia innominata, at least in the monkey, the responses of the neurons associated with gustatory stimuli occur when the animal is hungry and not when he is satiated (see above and ref. 3).

A hypothesis which is consistent with these findings is that some of these neurones in the lateral hypothalamus and substantia innominata which are activated by food in the hungry animal are involved in the reactions of the animal to food when he is hungry, that is to food reward ${ }^{24-28}$. According to the hypothesis, the reason why the animal works to obtain the sensory stimulation associated with, for example, the sight and taste of food only when he is hungry, is that this is when the sensory 
stimulation activates these neurones. The sensory stimulation would, by activating these neurones, provide the reward for which the animal worked. Brain-stimulation reward of this, and perhaps of some other regions would occur because in activating these neurones the stimulation mimics the effects which food has in the hungry animal on these neurones. Before this hypothesis can be accepted, evidence is needed on whether the activity of these neurones precedes feeding behaviour, or reflects feeding behaviour. To test this, the latency of activation of these hypothalamic neurones by the sight of food was measured, by presenting the food from behind a wide-aperture shutter. The latencies were in the range 150-200 msec. Further, it was shown that these neurones fired in a visual discrimination for food with latencies of 150-200 msec to the stimulus which signified that food was available, and did not respond to the stimulus which indicated that food was not available, and that latencies of the EMG associated with the lick responses were $200-350 \mathrm{msec}^{32}$. Thus the firing of the hypothalamic units preceded and predicted the responses of the hungry animal to food ${ }^{32}$. Also, the findings that brain-stimulation reward activates these neurones activated by food reward, and that self-stimulation occurs in the region of these neurones, suggest that the activity of the neurones could be involved in the reaction of the animal to the food reward, that is in the initiation and maintenance of feeding when food is available. It is now of importance to analyze the output connections of these neurones, to determine whether they could be involved in the effects produced by food reward such as the initiation of feeding behaviour, and the endocrine and autonomic responses which occur to food in the hungry animal.

If neurones in the forebrain which are related to brain-stimulation reward are concerned with processes of this type, then it need not be expected that there would be only one reward system, or that activation of these systems would be necessary for reward. Rather, brain-stimulation reward at some forebrain sites would occur because of activation of any one of a number of different systems concerned with responses to different natural reinforcers such as food or water $25,26,30$. At other self-stimulation sites there could be other substrates for the self-stimulation (see refs. 24 and 29).

\section{ACKNOWLEDGEMENTS}

Supported by the Medical Research Council.

\section{REFERENCES}

1 Bishop, M. P., Elder, S. T. and Heath, R. G., Intracranial self-stimulation in man, Science, 140 (1963) 394-395.

2 Briese, E. and Olds, J., Reinforcing brain stimulation and memory in monkeys, Exp. Neurol., 10 (1964) 493-508.

3 Burton, M. J., Rolls, E. T. and Mora, F., Effects of hunger on the responses of neurones in the lateral hypothalamus to the sight and taste of food, Exp. Neurol., 51 (1976) 668-677.

4 Delgado, J. M. R., New orientations in brain stimulation in man. In A. Wauquier and E. T. Rolls (Eds.), Brain-Stimulation Reward, North-Holland, Amsterdam, 1976, pp. 481-503.

5 Emmers, R. and Akert, K. A., A Stereotaxic Atlas of the Brain of the Squirrel Monkey, University of Wisconsin Press, Madison, 1963. 
6 German, D. C. and Bowden, D. M., Catecholamine systems as the neural substrate for intra-cranial self-stimulation: a hypothesis, Brain Research, 73 (1974) 381-419.

7 German, D. C. and Fetz, E. E., Responses of primate locus coeruleus and subcoeruleus neurons to stimulation at reinforcing brain sites and to natural reinforcers, Brain Research, 109 (1976) 497-514.

8 Grossman, S. P., Role of the hypothalamus in the regulation of food and water intake, Psychol. Rev., 82 (1975) 202-224.

9 Hamburg, M. D., Hypothalamic unit activity and eating behavior, Amer. J. Physiol., 220 (1971) 980-985.

10 Hubel, D., Tungsten micro-electrode for recording from single units, Science, 125 (1957) 549-550.

11 Ito, M., Mapping unit responses to rewarding stimulation. In A. Wauquier and E. T. Rolls (Eds.), Brain-Stimulation Reward, North-Holland, Amsterdam, 1976, pp. 89-95.

12 Mora, F., Rolls, E. T. and Burton, M. J., Modulation during learning of the responses of neurones in the lateral hypothalamus to the sight of food, Exp. Neurol., 53 (1976) 508-519.

13 Mora, F., Mogenson, G. J. and Rolls, E. T., Activity of neurones in the region of the substantia nigra during feeding, Brain Research, 133 (1977) 267-276.

14 Norgren, R., Gustatory responses in the hypothalamus, Brain Research, 21 (1970) 63-77.

15 Norgren, R., Taste pathways to hypothalamus and amygdala, J. comp. Neurol., 166 (1976) 17-30.

16 Olds, J., A preliminary mapping of electrical reinforcing effects in the rat brain, J. comp. physiol. Psychol., 49 (1956) 281-285.

17 Olds, J., Hypothalamic substrates of reward, Physiol. Rev., 42 (1962) 554-604.

18 Olds, J., Drives and Reinforcement, Raven Press, New York, 1977.

19 Plotnik, R., Mir, D. and Delgado, J. M. R., Map of reinforcing sites in the rhesus monkey brain, Int. J. Psychobiol., 2 (1972) 1-21.

20 Rolls, E. T., Involvement of brain stem units in medial forebrain bundle self-stimulation, Physiol. Behav., 7 (1971) 297-310.

21 Rolls, E. T., Contrasting effects of hypothalamic and nucleus accumbens septi self-stimulation on brain stem single unit activity and cortical arousal, Brain Research, 31 (1971) 275-285.

22 Rolls, E. T., Activation of amygaloid neurones in reward, eating and drinking elicited by electrical stimulation of the brain, Brain Research, 45 (1972) 365-381.

23 Rolls, E. T., The neural basis of brain-stimulation reward, Progr. Neurobiol., 3 (1974) 71-160.

24 Rolls, E. T., The Brain and Reward, Pergamon, Oxford, 1975.

25 Rolls, E. T., The neurophysiological basis of brain-stimulation reward. In A. Wauquier and E. T. Rolls (Eds.), Brain-Stimulation Reward, North-Holland, Amsterdam, 1976, pp. 65-87.

26 Rolls, E. T., Neurophysiology of feeding, Life Sci. Res. Rep. 2, 1976, pp. 21-42.

27 Rolls, E. T., Activity of hypothalamic and related neurons in the alert animal. In P. J. Morgane and J. Panksepp (Eds.), Handbook of the Hypothalamus, 3A, Dekker, New York, 1981.

28 Rolls, E. T., Neurophysiology of feeding, Trends Neurosci., 1 (1978) 1-3.

29 Rolls, E. T., Effects of electrical stimulation of the brain. In K. Connolly (Ed.), Psychology Survey No. 2, George, Allen and Unwin, London, 1979, pp. 151-169.

30 Rolls, E. T., Burton, M. J. and Mora, F., Hypothalamic neuronal responses associated with the sight of food, Brain Research, 111 (1976) 53-66.

31 Rolls, E. T. and Rolls, B. J., Activity of neurones in sensory, hypothalamic and motor areas during feeding in the monkey. In Y. Oomura (Ed.), Food Intake and Chemical Senses, Tokyo University Press, 1977, pp. 525-549.

32 Rolls, E. T., Sanghera, M. K. and Roper-Hall, A., The latency of activation of neurones in the lateral hypothalamus and substantia innominata during feeding in the monkey, Brain Research, 164 (1979) 121-135.

33 Routtenberg, A., Gardner, E. L. and Huang, Y. H., Self-stimulation pathways in the monkey, Macaca mulatta, Exp. Neurol., 33 (1971) 213-224.

34 Sem-Jacobsen, C. W., Depth-Electrographic Stimulation of the Human Brain and Behavior, Thomas, Springfield, Ill., 1968.

35 Sem-Jacobsen, C. W., Electrical stimulation and self-stimulation in man with chronic implanted electrodes. Interpretation and pitfalls of results. In A. Wauquier and E. T. Rolls (Eds.), BrainStimulation Reward, North-Holland, Amsterdam, 1976.

36 Snider, R. S. and Lee, J. C., A Stereotaxic Atlas of the Monkey Brain, University of Chicago Press, 1961.

37 Wauquier, A. and Rolls, E. T. (Eds.), Brain-Stimulation Reward, North-Holland, Amsterdam, 1976. 